British Journal of Nursing 2020; 29 (22) : S27-S30

\title{
Quality of life of family caregivers of patients with a stoma: A cross- sectional study from Iran
}

\author{
Rafiei, H. ${ }^{a}$ Rashvand, F.a, Malmir, S. ${ }^{\text {b }}$

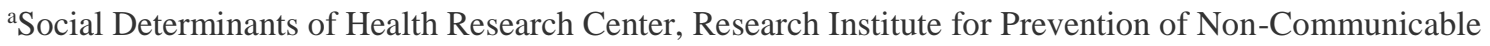 \\ Diseases, Qazvin University of Medical Sciences, Iran \\ ${ }^{b}$ Nursing Department, Faculty of Medical Science, Tarbiat Modares University, Tehran, Iran
}

Abstract

Aim:

The family plays a key role in supporting and caring for patients who have a gastrointestinal stoma because of cancer. This study investigated the quality of life of family members caring for such patients.

Methods:

A descriptive study was conducted in 2020 in Iran. The study sample consisted of 250 family members caring for cancer patients with an intestinal stoma. The Caregiver Quality of Life Index-Cancer scale was used to measure quality of life. This scale has 35 questions, which are categorised into four subscales: mental/emotional burden; lifestyle disruption; positive adaptation; and financial concerns. The highest possible total score is 140 ( 35 in each subscale), and a high score indicates a poor quality of life.

Results:

A total of 250 carers took part in the study. Of these, 143 (57.2\%) were men and the average age was 35.1 years. Mean scores of mental/emotional burden, lifestyle disruption, positive adaptation, and financial concerns were $28.4 \pm 41,17.3 \pm 3.2,19.8 \pm 28$, and $8.6 \pm 1.2$ respectively. No significant relationship was found between participants' quality of life and demographic variables.

Conclusion:

Carers' quality of life is relatively poor. Carers' quality of life need to be improved and support programmes, devised and delivered by nurses, could have a role in this. 